\title{
Correction for both common and rare cell types in blood is important to identify genes that correlate with age
}

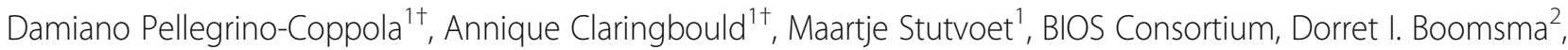
M. Arfan Ikram ${ }^{3}$, P. Eline Slagboom ${ }^{4}$, Harm-Jan Westra ${ }^{1}$ and Lude Franke ${ }^{1^{*}}$ (D)

\begin{abstract}
Background: Aging is a multifactorial process that affects multiple tissues and is characterized by changes in homeostasis over time, leading to increased morbidity. Whole blood gene expression signatures have been associated with aging and have been used to gain information on its biological mechanisms, which are still not fully understood. However, blood is composed of many cell types whose proportions in blood vary with age. As a result, previously observed associations between gene expression levels and aging might be driven by cell type composition rather than intracellular aging mechanisms. To overcome this, previous aging studies already accounted for major cell types, but the possibility that the reported associations are false positives driven by less prevalent cell subtypes remains.
\end{abstract}

Results: Here, we compared the regression model from our previous work to an extended model that corrects for 33 additional white blood cell subtypes. Both models were applied to whole blood gene expression data from 3165 individuals belonging to the general population (age range of 18-81 years). We evaluated that the new model is a better fit for the data and it identified fewer genes associated with aging $(625$, compared to the 2808 of the initial model; $\left.P \leq 2.5 \times 10^{-6}\right)$. Moreover, 511 genes ( $18 \%$ of the 2808 genes identified by the initial model) were found using both models, indicating that the other previously reported genes could be proxies for less abundant cell types. In particular, functional enrichment of the genes identified by the new model highlighted pathways and GO terms specifically associated with platelet activity.

Conclusions: We conclude that gene expression analyses in blood strongly benefit from correction for both common and rare blood cell types, and recommend using blood-cell count estimates as standard covariates when studying whole blood gene expression.

Keywords: Whole blood, Gene expression, Cell counts correction, Aging, Platelet activity

\footnotetext{
* Correspondence: ludefranke@gmail.com

${ }^{\dagger}$ Damiano Pellegrino-Coppola and Annique Claringbould contributed equally to this work.

'Department of Genetics, University Medical Centre Groningen, Groningen,

The Netherlands

Full list of author information is available at the end of the article
}

(c) The Author(s). 2021 Open Access This article is licensed under a Creative Commons Attribution 4.0 International License, which permits use, sharing, adaptation, distribution and reproduction in any medium or format, as long as you give appropriate credit to the original author(s) and the source, provide a link to the Creative Commons licence, and indicate if changes were made. The images or other third party material in this article are included in the article's Creative Commons licence, unless indicated otherwise in a credit line to the material. If material is not included in the article's Creative Commons licence and your intended use is not permitted by statutory regulation or exceeds the permitted use, you will need to obtain permission directly from the copyright holder. To view a copy of this licence, visit http://creativecommons.org/licenses/by/4.0/ The Creative Commons Public Domain Dedication waiver (http://creativecommons.org/publicdomain/zero/1.0/) applies to the data made available in this article, unless otherwise stated in a credit line to the data. 


\section{Background}

Aging, defined as a time-dependent process characterized by physical and cognitive decline, is one of the main risk factors for autoimmune diseases, neurodegenerative diseases, cancer and diabetes $[1,2]$. To better understand this process on a molecular level, changes in gene expression during aging have been previously studied in whole blood $[3,4]$. However, blood contains many cell populations, such as white blood cells (WBC) that can be divided into granulocytes, lymphocytes and monocytes, and further into more specific WBC subtypes [5]. Since the proportions of these cell populations vary with age [6-9], it is necessary to correct for cell counts when using gene expression from blood. Indeed, uncorrected gene expression data from whole blood has been shown before to be biased by the gene expression pattern of the most abundant cell type at the moment of sampling [10].

Here, to better identify cell-independent transcriptional signatures during aging, we expanded the regression model that corrects for the number of WBC presented in our previous work [3] (hereafter called Initial Model, IM), by taking into account additional specific WBC subtype counts in our new model (hereafter called Extended Model, EM). We compared the performance of these two models in a meta-analysis using
3165 human peripheral blood-derived RNA-seq samples from four independent Dutch cohorts present in the BIOS consortium, namely LifeLines Deep, Leiden Longevity Study, Netherlands Twin Registry and Rotterdam Study [11-14]. Further, we show that the EM complies with the assumptions of linear regression and provides a better fit to the data as residuals decrease. Lastly, we analyze the genes significantly up- and downregulated by functional enrichment in order to understand to which extent the models and cell correction can be used to extract biological information regarding aging in a general population.

\section{Results}

Improved cell correction is necessary to identify cellindependent gene expression patterns

We performed an association of gene expression changes with age using data from four Dutch cohorts (Table S1). To take into account the differences in the data, we conducted a meta-analysis across these cohorts. We included only samples with all categorical covariates reported, leaving a total of 3165 individuals (Table S1). An overview of this study is presented in Fig. 1.

We tested 19,932 genes expressed in blood and analyzed the data by applying two models, the IM and the

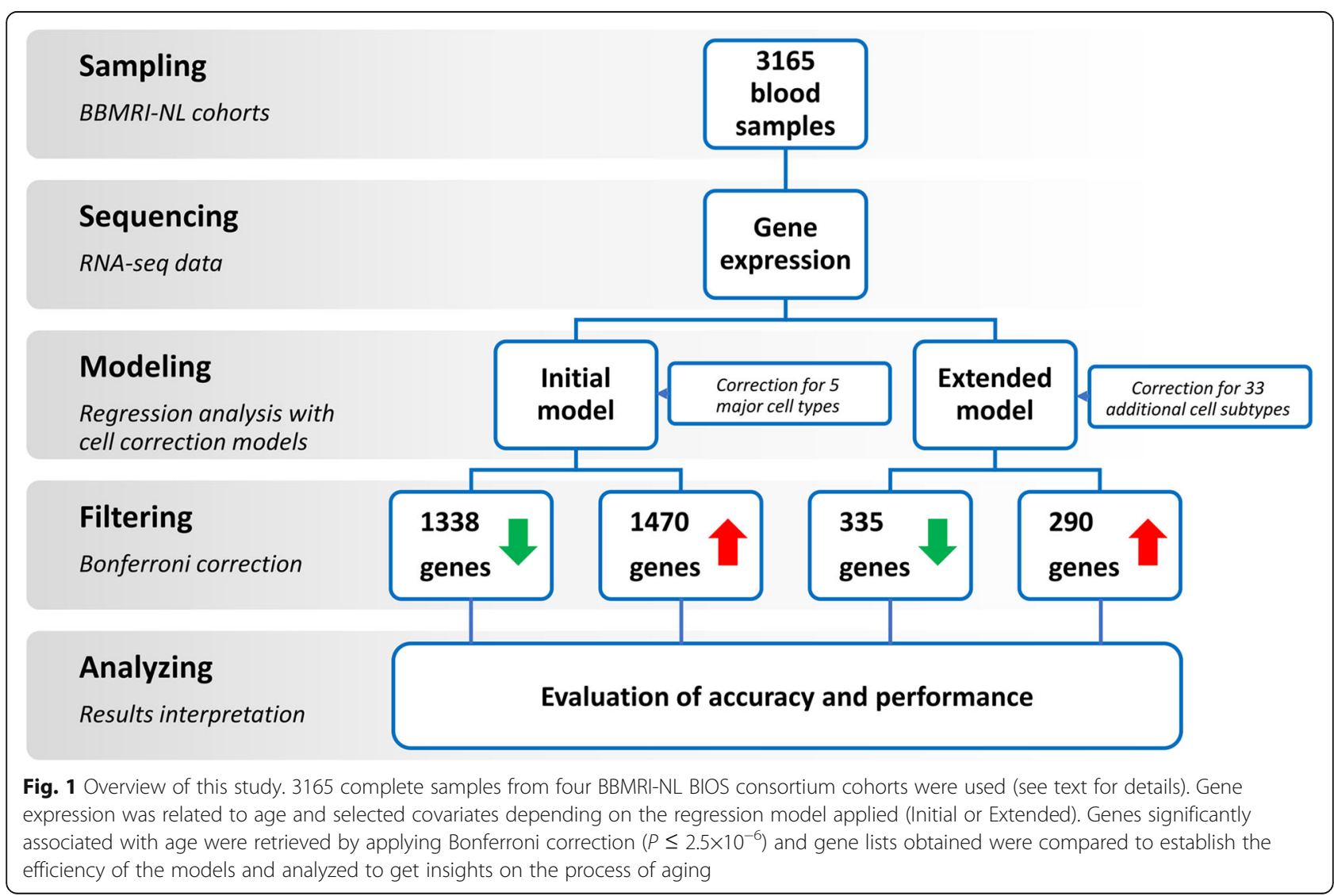


EM (see Methods and Fig. 1). The IM was presented previously [3]: it accounts for the main WBC types (number of granulocytes, lymphocytes, monocytes), erythrocytes and platelets, while our new model here presented, EM, corrects for 33 additional WBC subtypes (see Methods, Table S2 and S3, Fig. S1A). These additional WBC subtypes were imputed with Decon-cell [15]. We observed small but significant correlations between age and most measured and imputed cell counts (Fig. S1B), presenting evidence that adding WBC subtypes is beneficial for the correction models. For example, different imputed cell types, such as naïve $\mathrm{CD}^{+}$subtypes (IT50 and IT54 [16]), show a strong negative correlation with age when considering both the overall (data not shown) and the single cohorts (Fig. S1B).

Using the IM, we identified 1338 genes significantly downregulated and 1470 upregulated with age after Bonferroni correction $\left(P \leq 2.5 \times 10^{-6}\right)$ (Table S4 and Fig. 1). The EM, however, reduced the number of results substantially: we identified 335 downregulated and 290 upregulated genes significantly associated with aging at the same significance threshold (Table S4 and Fig. 1). This decrease was expected, as many of the results from the IM may have been driven by the composition of less prominent cell types that were included in our EM. While 511 out of 625 EM genes were also present in the IM results, the 114 additional EM genes were only detected after rigorous correction for cell types (Fig. S2). To validate our results, we compared the number of genes retrieved through our models with the 1497 genes reported in our previous work [3] (gene set 1, GS1) and the 481 genes identified by Lin and colleagues [4] (gene set 2, GS2), a study that uses a slightly different correction model to study aging. As reported in Table S5, the highest number of overlapping genes was found between the IM and the GS1 (672, 24\% of our 2808 IM genes). Considering that the number of tested genes is different (11,908 for GS1, based on a minimum level of expression across study cohorts, and 19,932 for IM, 10,890 in common), this overlap is quite large. Moreover, all genes had the same direction of association with age. These results are unsurprising, because we used the same previous correction model [3]. When comparing the EM results with the GS1, the number of overlapping genes decreased (172, 28\% of our EM genes) but the majority still had the same direction (98\%). The lowest number of overlapping genes was found between the EM results and the GS2 (9 genes overlapping, 7 with the same direction). In general, differences in the number of overlapping genes may result from: 1) differences in the model used, 2) differences in the technical analyses performed [17] and 3) differences between the genes used in the discovery phase. Overall, the models show a good conservation of direction for overlapping genes, which indicates that correcting for cell populations identifies common whole blood gene expression patterns.

\section{The extended model performs better than the initial model}

We next investigated whether both IM and EM met assumptions of linear regression. To this end, we analyzed the mean squared errors (MSE), the distribution of gene expression residuals and their homoscedasticity after applying the IM and EM. We first analyzed the impact of adding additional terms to our regression models on the MSE. As expected, MSE values of the regressions decreased when applying the EM (total EM median MSE value: 0.267 , total IM median MSE value: 0.334) (Fig. 2A-B and Table S6). We next created QQ-plots and calculated the Pearson correlation coefficient between the observed and expected distributions to assess normality. For most genes, including the 511 shared between IM and EM, we found that applying the EM resulted in more normally distributed residual values and the correlation values were higher (total EM median $r$ value: 0.995 , total IM median $\mathrm{r}$ value: 0.994) (Fig. 2C-D, Table S6 and S7). Lastly, we wanted to evaluate heteroskedasticity (i.e. the skewness on the distribution of residuals), as this can indicate a relation between the error and the explained variable, violating the model assumptions. For this purpose, we created a modified version of both models that included all covariates with the exception of age and applied the four resulting models (IM, EM, IM-age, EM-age) in each cohort. Then, we used the rank-based Spearman correlations to correlate gene expression residuals with age $[18,19]$. We checked the normality of these Spearman $\rho$ values and meta-analyzed them across the cohorts (Fig. S3A). We observed that the absolute correlations were smallest in the EM model (EM median value: $9 \times 10^{-3}$ ), and largest in the IM without age (IM-age median value: $6 \times 10^{-2}$ ) (Fig. $2 \mathrm{E}-\mathrm{F}$, Table S6 and S7 and Fig. S3B). Large $\rho$ values indicate a less precise prediction and larger errors. In general, the EM performs better than the IM, and it is specifically noteworthy that the EM without age performs better than the IM without age. Adding cell counts clearly improves the prediction of gene expression values. These three analyses indicate that the EM satisfies the assumptions of linear regression better than the IM. Moreover, adding cell counts as covariates improves reliable identification of aging-related genes in whole blood.

\section{Single-cell RNA-seq data reveals the contribution of cell types to gene expression during aging}

Every cell type has its own gene expression pattern, so the composition of blood cells influences the total gene expression observed in whole blood RNA-seq data. To test to which extent the aging-related genes found by 


\section{Analyses of Gene Expression Residuals}

A)
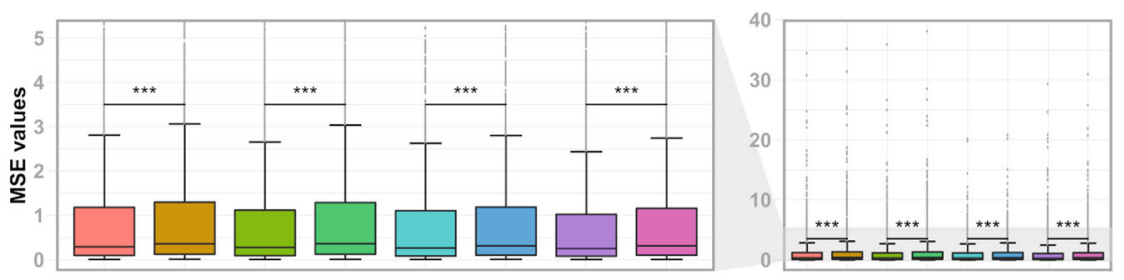

B)

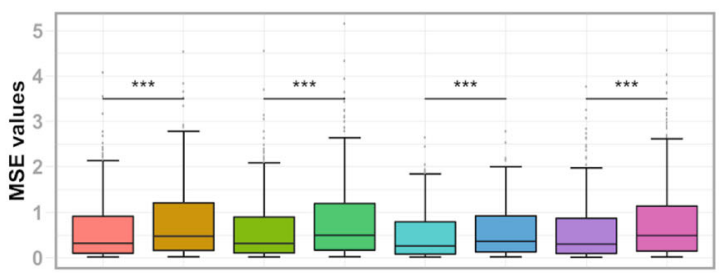

C)
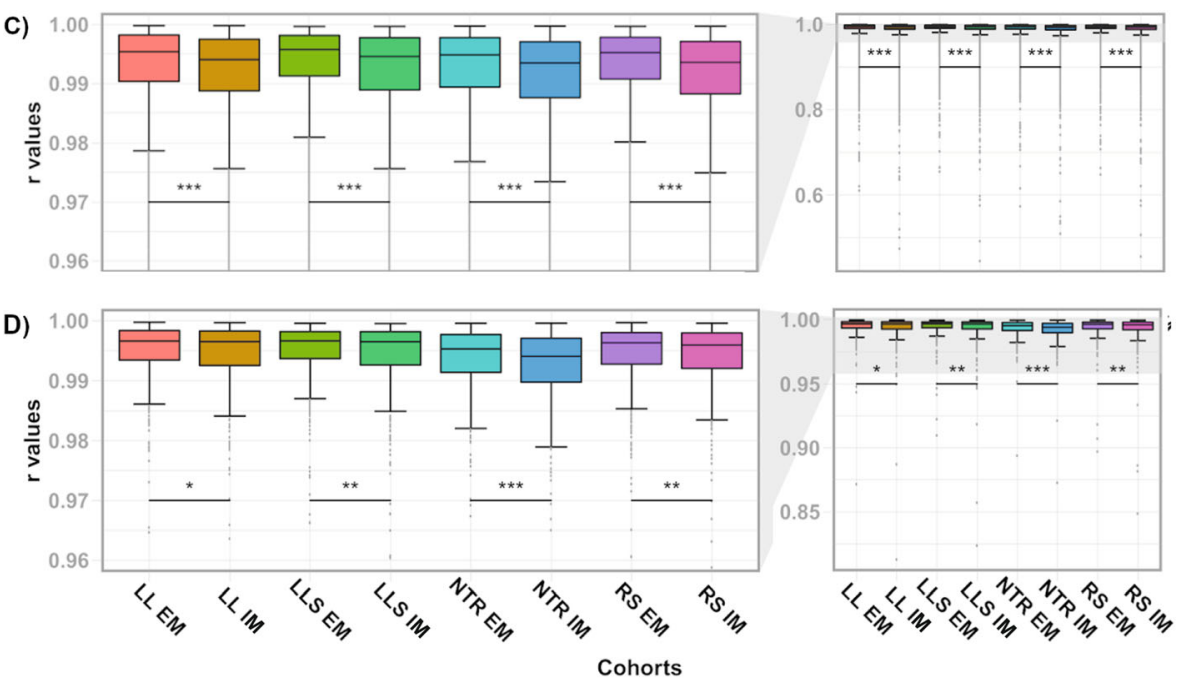

E)
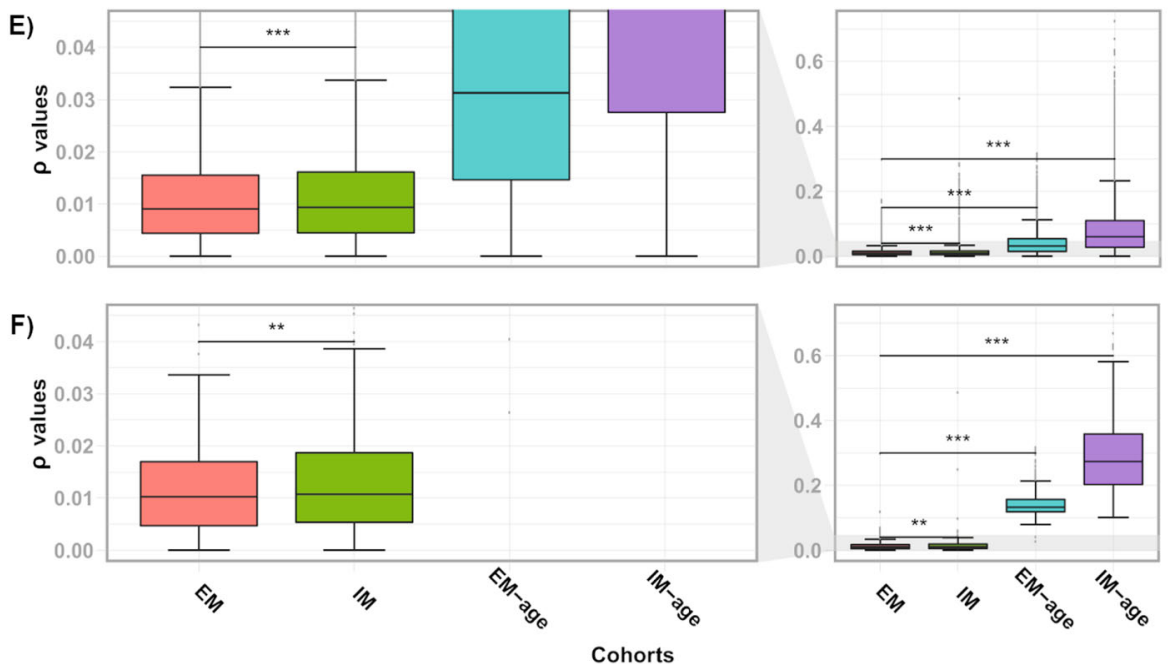

Fig. 2 (See legend on next page.) 
(See figure on previous page.)

Fig. 2 Gene expression residuals decrease with the EM. MSE values for regressions related to genes in every cohort after applying the IM and the EM are reported for all genes in (A), and the 511 shared genes in (B). QQ plot Pearson correlation coefficients ( $r$ values) related to the distributions of gene expression residuals are shown for all genes in (C) and for the shared genes significantly associated to aging in (D), after applying the IM and EM models. Homoscedasticity was evaluated by correlating gene expression residuals from every model with age, and the absolute Spearman $\rho$ values obtained after meta-analysis are reported for all genes (E) and the shared genes significantly associated with aging (F). LL, LifeLines DEEP; LLS, Leiden Longevity Study; NTR, Netherlands Twin Registry; RS, Rotterdam Study; EM, extended model; IM, initial model; EM-age, extended model without age as covariate; IM-age, initial model without age as covariate. Statistical significance was assessed with a paired, one-tailed Wilcoxon test. The stars indicate statistical significance: ${ }^{* *} P \leq 0.001,{ }^{* *} P \leq 0.01,{ }^{*} P \leq 0.05$

the models were influenced by blood cell populations, we investigated the mean expression of these agingrelated genes in single-cell RNA-seq (scRNA-seq) data of 11 different blood cell types [20]. As shown in the tSNE plots (Fig. 3A and B), aging-related genes retrieved through the IM have a propensity to be expressed in specific parts of the t-SNE plot that match with cell types, while EM genes maintain a lower and more stable expression across cell types from donors with a wide age range (Wilcoxon test, $P \leq 2.2 \times 10^{-16}$, Fig. $3 \mathrm{C}$ and $\mathrm{D}$ ), suggesting that it is not a specific cell type driving the associations. Secondly, we used differential expression patterns to identify blood cell type specific markers in the list of IM or EM significant aging-related genes, and visualized the mean expression in t-SNE plots (Fig. S4). The EM aging-related genes contain fewer cell type specific markers: no markers could be identified for three cell types (Natural Killer bright subset, $\mathrm{CD} 8^{+} \mathrm{T}$ and B cells). Importantly, the cell type marker genes that were identified among EM genes are less representative for their cell types than the IM markers, as shown in Fig. S4. In addition, we observed that the mean expression range for the EM genes was always larger, highlighting a higher gene expression variation (mean expression

\section{A)}

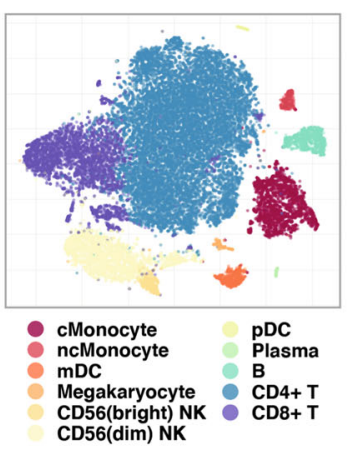

C)
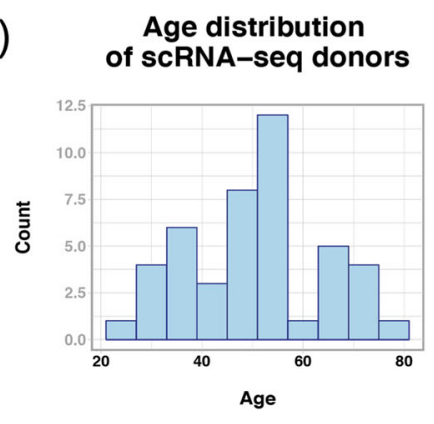

B)

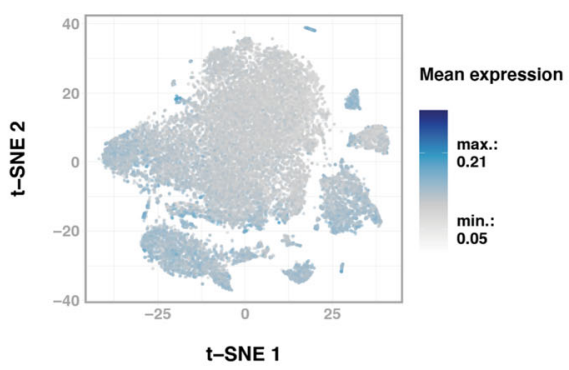

Initial model

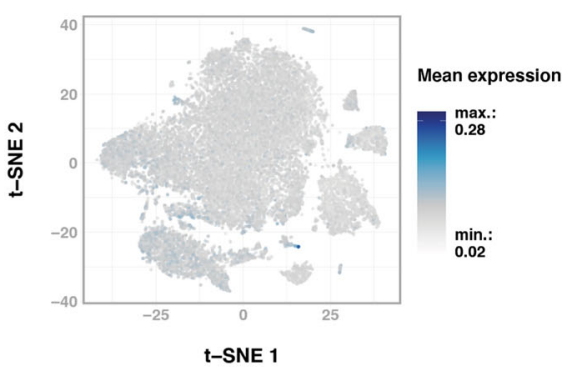

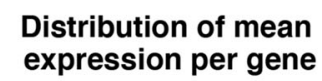

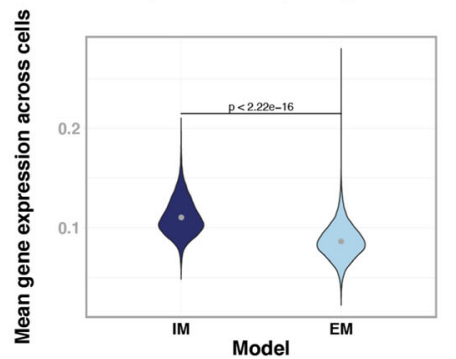

E) Distribution of expression variation per gene

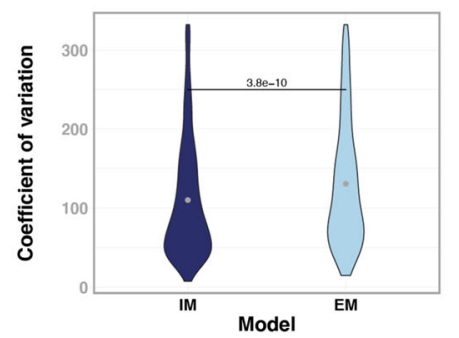

Fig. 3 EM aging-related genes are not related to specific blood cell populations. a) Cell-type clustering of single cell RNA-seq (scRNA-seq) data. b) The mean expression value of aging-related genes from the initial model (IM, left) and the extended model (EM, right) is plotted in the single cells. c) The age distribution of the scRNA-seq donors. In $\mathbf{d}$ ), the distributions of the mean gene expression for IM- and EM-related genes across every cell in the t-SNE plots from $\mathbf{b}$ ) are reported. e) The distributions of the coefficient of variation are presented for both the IM and EM. Statistical significance for $\mathbf{d}$ ) and e) was assessed with a Wilcoxon test. The level of statistical significance was set at $P \leq 0.05$. For details regarding cell population-specific regions, refer to [20] 
of IM genes per cell: 0.05-0.21; EM: 0.02-0.28, Fig. 3B and S4). This observation was supported by the scRNAseq coefficients of variation (Wilcoxon test, $P=$ $3.794 \times 10^{-10}$, Fig. 3E). In summary, the scRNA-seq data indicate that EM genes are less driven by cell types than the IM genes, suggesting that the EM model enables a better identification in blood of cell-quantity independent genes related to aging.

\section{Functional enrichment analysis and aging signatures}

In order to investigate whether the EM-derived agingrelated genes were more informative than the IMderived genes, we performed functional enrichment using Enrichr [21]. As 82\% of the EM genes were also present in the IM list, we expected comparable functional enrichments. On the contrary, very few pathways and GO terms were shared between the EM and IM lists (Table S8). The fact that we observed a smaller number of genes in the EM list did not translate to a lower number of EM-specific enrichments. Therefore, we hypothesized that although a high number of genes is shared between the EM and the IM, the difference in the functional enrichment results was due to the exclusion of genes that are influenced by cell quantity, for which the IM did not correct. Indeed, the enrichments for the EM genes clustered around potential aging-related mechanisms. For example, changes in GO biological processes ascribable to the regulation of gene expression were downregulated (e.g. 'regulation of transcription, DNA- templated' - GO:0006355, 'regulation of nucleic acidtemplated transcription' - GO:1903506, 'regulation of protein processing' - GO:0070613), in agreement with previous findings [3] and the IM results.

Hemostasis, the process to prevent and stop bleeding, emerged as a key upregulated pathway from the various EM-related enrichment analyses (Table S8). The KEGG pathway 'coagulation cascade' and the Reactome pathway 'hemostasis' were both significantly upregulated $(P$ $\leq 6.2 \times 10^{-4}, P \leq 4.5 \times 10^{-6}$, respectively), suggesting that changes in the expression of genes related to hemostasis and platelet functioning during aging have a very robust signature, as previously reported [22-26]. Changes in GO biological process terms related to platelet activity (GO:0045055, GO:0002576, Table S8) and GO cellular compartment terms linked to platelet granules (e.g. 'platelet alpha granule' - GO:0031091, Table S8) were also found to be significant. The EM-related genes driving these results are reported in Table S9. Notably, both models included the correction for platelet counts, suggesting that these functional enrichments described the activity of platelets independently of their prevalence. Platelet count remained more or less stable during aging in our data (Fig. S1B), so the number of platelets is not expected to drive these enrichments.

After applying the EM, we expected that genes involved in the same biological process and under the same regulation could show a common pattern. To identify this pattern, we calculated the correlations between

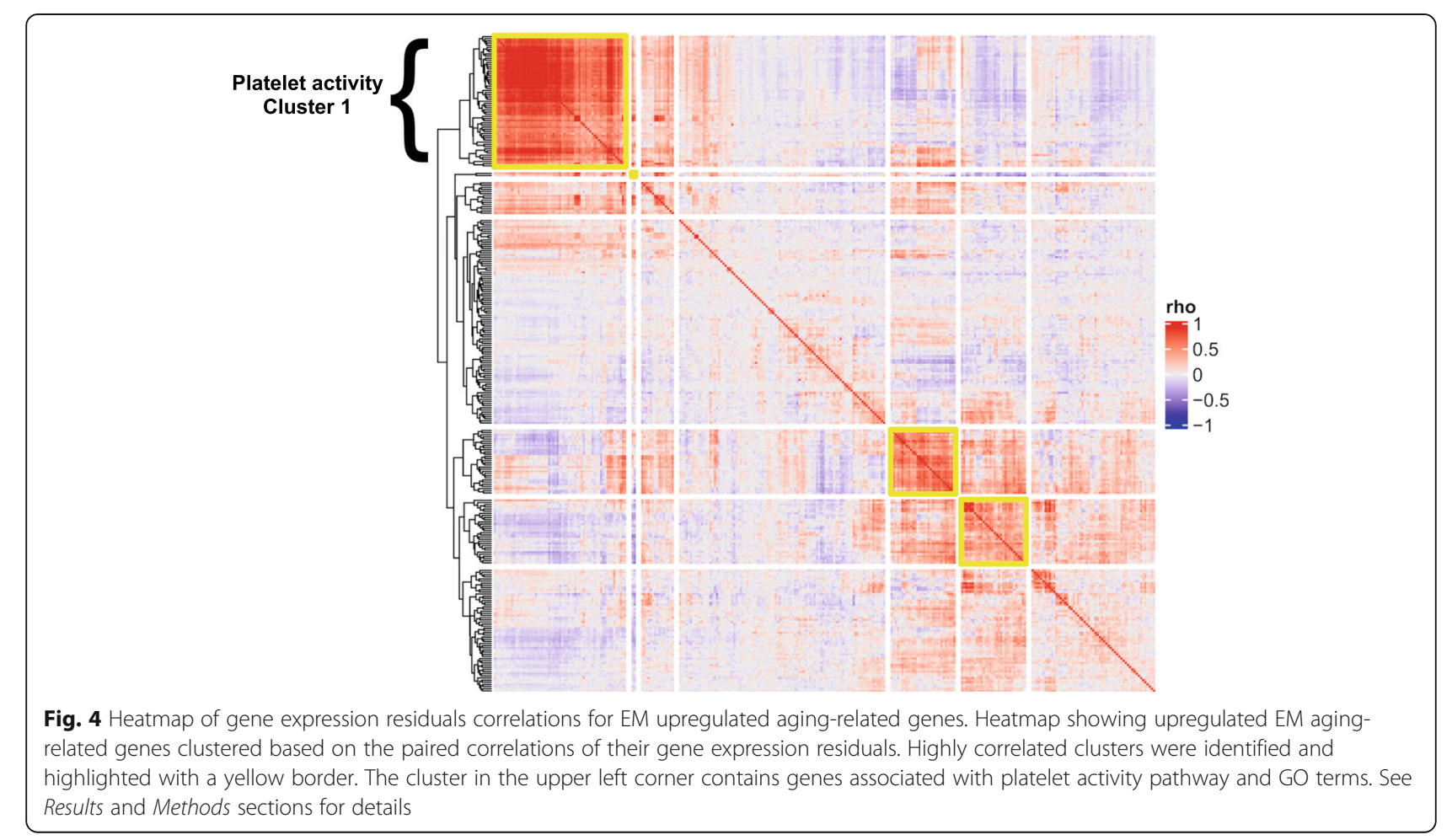


the gene expression residuals. We observed several clusters with highly correlating values (Fig. 4 and Fig. S5), which we further analyzed with Enrichr. While most clusters did not show a clear enrichment, cluster 1 of the upregulated EM aging-related genes (Fig. 4, upper left corner) was enriched for terms related to platelet activity, again highlighting its role in aging (Fig. S6). Five genes (PF4, PPBP, STON2, MYLK, LMNA) from the platelet-related cluster 1 were previously identified to be differentially expressed with age in platelets [25]. Although PF4 and PPBP did not show the same direction of effect, a difference that may result from the sample size or the model used, the overall finding that platelets show increased activity with age is conserved [22, 23, 25, 26]. The EM enrichment result of platelet activity was independent of the measured number of platelets. The correlations between gene expression levels of the genes from cluster 1 that contribute to the enrichment and measured platelet levels are very significant (Fig. $\mathrm{S} 7 \mathrm{~A}$ ), but they disappear when we compare the residual gene expression from the EM with such platelet counts (Fig. S7B).

Lastly, we explored the GenAge database [27] as a resource to identify enrichments in aging genes when considering both IM or EM results. Both IM- and EMrelated gene lists were found to contain aging genes reported also in GenAge (Table S10). In particular, all the EM-related genes identified as aging genes in GenAge were found to be a subset of the IM-related genes, with the exception of EMD coding for emerin. This result is particularly intriguing as EMD has a role in nuclear lamina, already known for its relevance in aging through LMNA. In addition, mutations in both EMD and LMNA are involved in the Emery-Dreifuss muscular dystrophy. This result highlights once more the filtering properties of the EM model, and further suggests its ability in making aging information stand out.

\section{Discussion}

Aging is a process that enhances the probability of getting diseases such as cancer, diabetes and various types of neurodegeneration. In order to understand how an organism reaches these diseased states, it is valuable to study the preceding period, where the organism ages. Changes can be investigated by analyzing aging cohorts as representatives of an aging population. Following this reasoning, in this study we used four Dutch aging cohorts (Table S1) and analyzed gene expression changes during aging in whole blood, an easily accessible tissue, by implementing a new model (EM) to correct for cell type proportions. This extended cell correction enabled us to calibrate gene expression according to the number of blood cells and extract an aging gene expression pattern that was less influenced by cell quantity compared to previously published models $[3,4]$. The rationale behind the method we propose is that both variations in organismal cell composition and gene expression influence the processes of aging and diseases, and that cell correction enables to filter out the expression of specific cell biomarkers while aiming at retaining those gene expression patterns that capture the main and shared aging processes in the whole tissue. In turn, since aging is known to be tightly linked with diseases, the analyses presented here could form a starting point to identify blood-related aging and disease players.

To test the performance of our EM, we evaluated its compliance to the assumptions of regression. The EM outperformed the old model, IM, when analyzing the MSE, normality of residuals and homoscedasticity, highlighting that an increased cell correction results in a more accurate gene expression estimation during aging.

Next, we asked which cell population contributed the most to the list of aging-related genes provided by both the IM and EM. For this purpose, we calculated per cell type the mean gene expression of both IM and EM genes using scRNA-seq data from $~ 25,000$ blood mononuclear cells of 45 donors [20]. The EM aging-related genes had lower mean gene expression levels, fewer cell type specific marker genes and those markers that were present were less abundantly expressed (Fig. 3 and S4). We consequently reasoned that these genes are less influenced by cell composition and quantity.

We performed a functional enrichment analysis for GO terms, KEGG and Reactome pathways in order to gain insight on the blood-based biological mechanisms driving aging. Although many of the EM genes were also identified using the IM, the enrichments were often not overlapping suggesting an increased precision in evaluating the relation between gene expression and age. In particular, platelet-related categories stood out in these results. We clustered the EM genes based on gene expression residuals and again found the strongest enrichment in the upregulation of platelet activity.

Since our EM includes a correction for platelet counts, the observation that platelet activation is enriched in relation to the EM aging-related genes is possibly due to the following reasons: 1) the EM did not correct for cell counts sufficiently or 2) an increase in platelet activity is a true signature of aging. While we cannot exclude the first reason, the fact that platelets do not associate with age in our data make it less plausible. We also show that there is no residual relationship between platelet counts and gene expression after correction (Fig. S7B). Moreover, platelet activity has been reported to increase with age in literature [22, 23, 25, 26] and incubating human platelets with media from senescent human fibroblasts increases platelet activation and degranulation [24]. Upon degranulation, platelets release the factors present 
in their granules into the surrounding environment. Of note, our functional enrichment analysis retrieved GO terms related to alpha granules, which store PPBP and PF4. These proteins are known to be increasingly secreted during aging [22, 28, 29]. The genes encoding these proteins were found to be upregulated agingrelated genes and, more specifically, they contributed to the enrichment of alpha-granule-related cell compartment GO terms (Table S8). Interestingly, an earlier study that performed RNA-seq within isolated platelets has observed decreased expression of PF4 and PPBP with age $(n=154$ [25]), while studies in whole blood show upregulation with age (current study: both genes significant; in the previous study [3]: PF4 not tested, $P P B P$ nominally significant). Within our scRNA-seq data, both genes are specifically expressed in megakaryocytes, the precursors of platelets (Fig. S8), suggesting that the observed upregulation is not driven by the expression in any other blood cell types, but by platelets or megakaryocytes themselves. Although these results may arise from differences in sample sizes or models used, this observation coupled with the fact that older individuals have higher levels of PF4 and PPBP protein in their plasma indicates that platelets become more active with age as reflected both in gene expression levels and protein abundance in plasma [22].

In addition, alpha granules are known to store agingrelated proteins, such as IGF1, a protein that has been extensively connected to aging together with its orthologs in multiple organisms [30, 31]. Therefore, an enhanced platelet degranulation itself, as a consequence for instance of an increased signaling by senescent cells, could have a major impact on the progression of aging, pointing at platelet activity as an aging hallmark and biomarker.

\section{Conclusions}

Overall, we have shown that an extensive correction for cell type differences can dramatically alter the effect sizes and significance of associations between genes and age. On top of this correction for measured or imputed cell counts, we believe that large scRNA-seq datasets (e.g. sceQTLGen consortium [32], The Human Cell Atlas [33]) will be essential to visualize and quantify to what extent associations are independent of cell type composition and how individual cell populations change with age. Our and previous findings [25] indicate that it will be essential to investigate to what extent the increased platelet activity is driven by megakaryocytes using larger blood-based scRNA-seq datasets [34]. Lastly, while the current study was performed in blood, other tissues also feature cell type heterogeneity. As such, we conclude that rigorous correction for cell type counts is important for studies in whole blood, and will help to better understand immune aging and other gene expression association studies. In summary, we hypothesize that the platelet enrichment observed in the EM aging-related genes represents one of the molecular signatures of aging. The increased platelet activation and subsequent release of aging factors could affect other cells and in turn the whole organism. However, many details regarding the mechanisms that are affected by these aging factors remain to be discovered.

\section{Methods}

\section{Study populations}

We performed a meta-analysis using 3165 human peripheral blood samples obtained from four independent Dutch cohorts: LifeLines DEEP (LL, $n=1100$ ) [11], Leiden Longevity Study (LLS, $n=585$ ) [12], Netherlands Twin Registry (NTR, $n=852$ ) [13] and Rotterdam Study (RS, $n=628$ ) [14] with participants from a wide age range (Table S1). None of the cohorts use disease as a selection criterion. LL participants are all from the Northern three provinces of the Netherlands, LLS includes the offspring and partners of long-lived individuals, NTR studies twins and their relatives and RS participants are all over 45 years old. All cohorts followed similar protocols for genotyping and gene expression as part of the BIOS Consortium, an initiative of the Biobanking and Biomolecular Resources Research Infrastructure - The Netherlands [35].

\section{Gene expression}

Gene expression data was obtained using the same protocol across all studies, as previously described [36]. Briefly, RNA was extracted from whole blood using PAXgene Blood miRNA Kit (Qiagen, California, USA) and paired-end sequenced with the Illumina HiSeq 2000 platform. After quality control by FastQC, adapters were removed and read quality trimming steps executed. Reads were aligned with STAR using GRCh37 as a reference while masking common (MAF $>1 \%$ ) SNPs in the Genome of the Netherlands [37]. Reads were assigned to genes with HTseq using gene definitions from Ensembl v71. Subsequently, expression values for all exons of each gene were added up to represent gene expression, measured in base count per gene. Prior to normalization, population outliers were removed based on a plot of the first two principal components, calculated on nonimputed genotypes. The first step in the normalization procedure was the application of the trimmed mean of M-values normalization method [38]. Next, we removed genes with no variance, $\log _{2}$ transformed the expression matrix and Z-transformed by centering and scaling of the genes, following a previously published protocol described in detail in the online cookbook [39]. 


\section{Cell count imputation}

We then performed imputation of cell counts, since these were not present for all included samples. For imputation, we only considered samples where all categorical covariates (sex, smoking status, fasting before blood sampling, RNA plate) were available (see Table S2 for missing values). We estimated the $33 \mathrm{WBC}$ subtypes included in the EM using the $\mathrm{R}$ package Decon-cell, a method that quantifies cell types using expression of marker genes (Table S3) [15]. The red blood cell (RBC) count was imputed using multivariate imputation by chained equations (MICE) from the $\mathrm{R}$ package MICE version 2.30 [40] because this cannot be imputed based on gene expression values, but rather relies on the other cell type counts and other phenotypes (Table S2). In MICE, we used predictive mean matching, as it has the advantage of imputing missing values within the observed spectrum after creating a normal distribution [40, 41]. Values outside the range of \pm 3 standard deviations from the mean were removed after $\log _{2}$ transformation.

\section{Models for differential expression during chronological age}

The IM was taken from the previous work [3] and is:

$$
y_{i} \approx \beta_{0}+\beta_{1} a g e_{i}+\beta_{2} x_{i 2}+\ldots+\beta_{p} x_{i p}+\varepsilon_{i}
$$

with y being gene expression levels for every gene, $i$ the number of cohort samples, age $\left(x_{i 1}\right)$ in years at time of blood sampling, and the following additional variables being the other covariates, including cell counts (for a total of $p$ predictors). Since we included 3165 samples, we have many more observations than predictors in the models $(I M=11, E M=44)$, suggesting that the models do not suffer from overfitting [42]. As covariates, we included sex, smoking status, fasting before blood sampling, RNA plate and GC content (an RNA-sequencing quality control score). All covariates were fixed effects, except for RNA plate, which was set as a random effect. As cell counts, we included the number of RBCs, platelets, granulocytes, lymphocytes and monocytes (Table S1). In our EM, the imputed proportions of $33 \mathrm{WBC}$ subtypes were included, to increase the power to detect cell-independent age effects. For a complete overview of WBC subtypes see Table S3. Both the IM and EM were tested on 19,932 genes that showed expression in blood of at least 0.5 counts per million in at least $1 \%$ of the samples [43]. For these tests, we used the lmer function from the R package lme4 version 1.1.13 [44]. Sample sizes, effect directions, and $P$-values were extracted from the result files of both linear models.

\section{Meta-analysis}

To combine associations across the four cohorts and to avoid bias of results due to cohort-specific effects, we first analyzed each cohort separately and then conducted a meta-analysis. We used the meta-analysis tool for genome-wide association scans (METAL) to calculate weighted Z-scores and $P$-values for every gene [45]. Although originally developed for meta-analysis of genome wide association studies, METAL was easily adapted for expression associations as described in the previous work [3].

\section{Evaluation of the regression models}

To evaluate the performance of the regression models, we used gene expression residuals and investigated MSE values, distribution of residuals and homoscedasticity. The distribution of residuals was evaluated by calculating the QQ plot Pearson correlation coefficient from sample and theoretical quantiles, considering that the higher the correlation value, the more the distribution approximates normality. Regarding homoscedasticity, meta-analysis was conducted on cohort-related, genespecific Spearman $\rho$ values (rho values) obtained by correlating age with the gene expression residuals, calculated from the application of the IM and EM. For this purpose, a Fisher Z-transformation was applied to the $\rho$ values after evaluating the approximation of their distribution to normality with a QQ plot. Then, Z-scores were combined across the cohorts using a weighted approach as described in [46] and the overall Z-score converted to $\rho$ with the inverse Fisher transformation.

\section{Functional enrichment analysis}

To better understand gene function, we performed functional enrichment using Enrichr [21]. For this analysis, we grouped genes significantly associated with aging $(P$ $\leq 2.5 \times 10^{-6}$, Bonferroni correction: 0.05/19,932 investigated genes) in either the IM or the EM into up- and downregulated genes. Using this approach, we retrieved information regarding enrichment in pathways based on KEGG and Reactome or GO terms. In addition, gene expression residuals were used to correlate genes significantly associated with aging. Correlating clusters were obtained with complete-linkage clustering and highly correlating clusters marked with a yellow border were identified by summing the correlation values within such cluster above 0 , and dividing this sum for the total number of correlations in that cluster, taking into account that a cluster with perfect correlations has only $1 \mathrm{~s}$, and since their sum would be identical to the total number of correlations, division would yield 1 (our threshold was set to 0.95$)$. 


\section{Single-cell RNA-seq data and visualization}

To interpret the cell type specificity of our ageassociated genes, we used scRNA-seq data for approximately $\sim 25,000$ peripheral blood mononuclear cells from $45 \mathrm{LL}$ donors. Collection and normalization of the data has been described previously [20]. We used the $\mathrm{R}$ package Seurat version 1.4.0.13 for scRNA-seq analyses and visualizations [47]. ScRNA-seq data enabled the detection of eleven cell types: classical and non-classical monocytes, myeloid and plasmacytoid dendritic cell, CD56 bright and dim natural killer cells, $\mathrm{CD}_{4}^{+}$and $\mathrm{CD}^{+} \mathrm{T}$ cells, $\mathrm{B}$ cells, plasma cells and megakaryocytes [20]. Within these cell types, we calculated the mean expression of the genes significantly associated with aging identified by the IM and the EM, and represented their expression in $\mathrm{t}-\mathrm{SNE}$ plots. We then identified genes that we considered markers for each of the 11 cell types using the function 'FindMarkers()' from Seurat using the loose thresholds of min.pct $=0.5$, min.diff.pct $=0.2$ to evaluate whether the aging-related genes were reflecting specific cell types.

\section{Supplementary Information}

The online version contains supplementary material available at https://doi. org/10.1186/s12864-020-07344-w.

Additional file 1: Table S1. Samples for which all covariates were available and were included in the analyses. LL, LifeLines DEEP; LLS, Leiden Longevity Study; NTR, Netherlands Twin Registry; RS, Rotterdam Study; IQ, interquartile range; $\mathrm{BMI}$, body mass index; $\mathrm{RBC}$, red blood cells; GC, guanine - cytosine. A RBC count was imputed for all LLS samples (see Methods)

Additional file 2: Table S2. Missing values expressed in number and percentage per cohort. Values are number (n) and percentage (\%). LL, LifeLines DEEP; LLS, Leiden Longevity Study; NTR, Netherlands Twin Registry; RS, Rotterdam Study.

Additional file 3: Table S3. Overview of all 33 imputed white blood cell (WBC) subtypes. The codes of each subtype used in the EM model are reported.

Additional file 4: Table S4. Summary statistics of gene associations with age for the initial model (this sheet) and the extended model (next sheet).

Additional file 5: Table S5. The number of total overlapping genes (upper part), the conservation of direction (middle part) and the percentage of genes associated with aging considering the discovery genes (lower part) is reported. EM, Extended Model; IM, Initial Model; GS1, gene set 1 from [3]; GS2, gene set 2 from [4].

Additional file 6: Table S6. Mean squared errors, Pearson correlation coefficient $(r)$ and Spearman correlation coefficient (rho) of residual gene expression with age. LL, LifeLines DEEP; LLS, Leiden Longevity Study; NTR, Netherlands Twin Registry; RS, Rotterdam Study; EM, extended model; IM, initial model; EM-age, extended model without age as covariate; IM-age, initial model without age as covariate.

Additional file 7: Table S7. Correlation coefficients per gene; initial model (im); extended model (em).

Additional file 8: Table S8. Enrichment of Reactome pathways, KEGG pathways and GO terms.

Additional file 9: Table S9. Overlap of IM- and EM-related genes with platelet-related genes derived from the GO, KEGG and Reactome enrichments described in Table S8.
Additional file 10: Table S10. Overlap of IM- and EM-related genes with the known aging-related genes in the GenAge database.

Additional file 11: Figure S1A. Heatmap of extended model predictors. A heatmap of Spearman correlations between all cell type predictors used in the extended model (EM). Figure S1B. Correlations of selected variable counts with age. The Spearman correlations of selected variables - including measured or imputed cell counts - with age are presented, colored per cohort (see legend). See Results section for details. Figure S2. Correlation of Z-scores associated with IM and EM genes. A Pearson correlation of the Z-scores associated with both significant and not significant IM and EM genes is shown. The $45^{\circ}$ diagonal is presented as dashed, the correlation line is in red. See Results section for details. Figure S3. Cohort-related, gene-specific $\rho$ values. A) QQ plots used to evaluate the distribution pattern of cohort-related, gene-specific $\rho$ values. B) Gene expression residuals decrease with the EM. Homoscedasticity was evaluated by correlating gene expression residuals from every model with age, and the absolute Spearman values obtained after metaanalysis are reported for all genes minus the shared genes significantly associated with aging. See Figure 2 in the main text and Methods for details. Statistical significance was assessed with a paired, one-tailed Wilcoxon test. The stars indicate statistical significance: ${ }^{* *} P \leq 0.001,{ }^{* *} P \leq$ 0.01 , $P \leq 0.05$. LL, LifeLines DEEP; LLS, Leiden Longevity Study; NTR, Netherlands Twin Registry; RS, Rotterdam Study; EM, extended model; IM, initial model; IM no age, IM without age as covariate; EM no age, EM without age as covariate. Figure S4. sCRNA-seq data-derived t-SNE plots reveal that IM-related aging genes are more likely cell type-specific marker genes. Mean expression levels of cell type marker genes among aging-related genes identified in the Initial Model (IM, left) and in the Extended Model (EM, right) are plotted. Where applicable, IM- and EMrelated intensities for same cell types plots were compared through a Wilcoxon test, always observing a $P \leq 2.2 \times 10^{-16}$. For details regarding cell population-specific regions, refer to [20]. Figure S5. Heatmap of gene expression residuals correlations for EM downregulated aging-related genes. Downregulated EM aging-related genes were clustered based on the correlations of gene expression residuals and highly correlating clusters were identified and highlighted with a yellow border. See Results section for details. Figure S6. Expression values of platelet-related genes across age. The four cohorts are colored separately and the Spearman correlations and $P$-values are calculated for each cohort independently. Figure S7. Correlations between (residual) gene expression levels of the genes from platelet-related cluster 1 and measured platelet levels. A) Spearman correlations between gene expression levels and measured platelets, B) Spearman correlations between gene expression residuals from the extended model and measured platelets. Figure S8. scRNA-seq data-derived t-SNE plots reveal that PF4 and PPBP are specifically expressed in megakaryocytes. Mean expression levels of platelet marker genes PF4 and PPBP are plotted. For details regarding cell population-specific regions, refer to [20].

\section{Abbreviations}

WBC: white blood cells; IM: Initial Model; EM: Extended Model; GS1: gene set 1; GS2: gene set 2; MSE: mean squared errors; IM-age: IM without age; EMage: EM without age; scRNA-seq: single-cell RNA-seq; LL: LifeLines DEEP; LLS: Leiden Longevity Study; RS: Rotterdam Study; NTR: Netherlands Twin Registry; RBC: red blood cell; MICE: multivariate imputation by chained equations; METAL: meta-analysis tool for genome-wide association scans

\section{Acknowledgements}

We thank the UMCG Genomics Coordination Center, MOLGENIS team, the UG Center for Information Technology, the UMCG research IT program and their sponsors in particular BBMRI-NL for data storage, high performance compute and web hosting infrastructure.

\section{Authors' contributions}

DPC and AC contributed equally to this work. DPC, AC and MS analyzed and interpreted the data. DPC and AC wrote the manuscript and created the figures. DIB, MAI, PES have contributed to the acquisition of the data and revised the manuscript. HJW contributed to the design and interpretation of the work and substantively revised it. LF made substantial contributions to data acquisition as well as the conception, design, interpretation and 
revision of the work. All authors have approved the submitted version and have agreed to be personally accountable for their own contributions.

\section{Authors' information}

Information on the members of the BIOS consortium, their role in the consortium and their affiliation is described as follows:

Management Team: Bastiaan T. Heijmans (chair)', Peter A.C. 't Hoen², Joyce van Meurs ${ }^{3}$, Aaron Isaacs ${ }^{4}$, Rick Jansen 5 , Lude Franke ${ }^{6}$

Cohort collection: Dorret I. Boomsma ${ }^{7}$, René Pool ${ }^{7}$, Jenny van Dongen? Jouke J. Hottenga ${ }^{7}$ (Netherlands Twin Register); Marleen MJ van Greevenbroek ${ }^{8}$, Coen D.A. Stehouwer ${ }^{8}$, Carla J.H. van der Kallen ${ }^{8}$, Casper G. Schalkwijk ${ }^{8}$ (Cohort study on Diabetes and Atherosclerosis Maastricht); Cisca Wijmenga ${ }^{6}$, Lude Franke ${ }^{6}$, Sasha Zhernakova ${ }^{6}$, Ettje F. Tigchelaar ${ }^{6}$ (LifeLines Deep); P. Eline Slagboom', Marian Beekman', Joris Deelen', Diana van Heemst $^{9}$ (Leiden Longevity Study); Jan H. Veldink ${ }^{10}$, Leonard H. van den Berg $^{10}$ (Prospective ALS Study Netherlands); Cornelia M. van Duijn ${ }^{4}$, Bert A. Hofman ${ }^{11}$, Aaron Isaacs ${ }^{4}$, André G. Uitterlinden ${ }^{3}$ (Rotterdam Study). Data Generation: Joyce van Meurs (Chair) ${ }^{3}$, P. Mila Jhamai ${ }^{3}$, Michael Verbiest ${ }^{3}$, H. Eka D. Suchiman', Marijn Verkerk ${ }^{3}$, Ruud van der Breggen', Jeroen van Rooij ${ }^{3}$, Nico Lakenberg' ${ }^{1}$.

Data management and computational infrastructure: Hailiang Mei $\left({ }^{(C h a i r}\right)^{12}$, Maarten van Iterson ${ }^{1}$, Michiel van Galen ${ }^{2}$, Jan Bot ${ }^{13}$, Dasha V. Zhernakova ${ }^{6}$, Rick Jansen ${ }^{5}$, Peter van 't Hof ${ }^{12}$, Patrick Deelen ${ }^{6}$, Irene Nooren ${ }^{13}$ Peter A.C. 't Hoen', Bastiaan T. Heijmans', Matthijs Moed'.

Data Analysis Group: Lude Franke (Co-Chair) ${ }^{6}$, Martijn Vermaat ${ }^{2}$, Dasha V. Zhernakova ${ }^{6}$, René Luijk ${ }^{1}$, Marc Jan Bonder ${ }^{6}$, Maarten van Iterson ${ }^{1}$, Patrick Deelen ${ }^{6}$, Freerk van Dijk ${ }^{14}$, Michiel van Galen ${ }^{2}$, Wibowo Arindrarto ${ }^{12}$, Szymon M. Kielbasa ${ }^{15}$, Morris A. Swertz ${ }^{14}$, Erik. W van Zwet ${ }^{15}$, Rick Jansen ${ }^{5}$, Peter-Bram 't Hoen (Co-Chair)', Bastiaan T. Heijmans (Co-Chair)'.

1. Molecular Epidemiology Section, Department of Medical Statistics and Bioinformatics, Leiden University Medical Center, Leiden, The Netherlands. 2. Department of Human Genetics, Leiden University Medical Center, Leiden, The Netherlands.

3. Department of Internal Medicine, ErasmusMC, Rotterdam, The Netherlands. 4. Department of Genetic Epidemiology, ErasmusMC, Rotterdam, The Netherlands.

5. Department of Psychiatry, VU University Medical Center, Neuroscience Campus Amsterdam, Amsterdam, The Netherlands.

6. Department of Genetics, University of Groningen, University Medical Centre Groningen, Groningen, The Netherlands.

7. Department of Biological Psychology, VU University Amsterdam, neuroscience campus. Amsterdam, Amsterdam, The Netherlands. 8. Department of Internal Medicine and School for Cardiovascular Diseases (CARIM), Maastricht University Medical Center, Maastricht, The Netherlands. 9. Department of Gerontology and Geriatrics, Leiden University Medical Center, Leiden, The Netherlands.

10. Department of Neurology, Brain Center Rudolf Magnus, University Medical Center Utrecht, Utrecht, The Netherlands.

11. Department of Epidemiology, ErasmusMC, Rotterdam, The Netherlands. 12. Sequence Analysis Support Core, Leiden University Medical Center, Leiden, The Netherlands.

13. SURFsara, Amsterdam, the Netherlands.

14. Genomics Coordination Center, University Medical Center Groningen, University of Groningen, Groningen, the Netherlands.

15. Medical Statistics Section, Department of Medical Statistics and Bioinformatics, Leiden University Medical Center, Leiden, The Netherlands

\section{Funding}

This work is supported by a grant from the European Research Council (ERC, ERC Starting Grant agreement number 637640 ImmRisk) to LF and a VIDI grant (917.14.374) from the Netherlands Organization for Scientific Research (NWO) to LF. LF is supported by a Senior Investigator grant from the Oncode Institute.

\section{Availability of data and materials}

The data that support the findings of this study are available from BBMRI-NL but restrictions apply to the availability of these data, which were used under license for the current study, and so are not publicly available. Raw RNA-seq data of the cohorts analyzed in this study can be obtained from the European Genome-Phenome Archive (study accession EGAS00001001077, dataset accession EGAD00001003785, https://www.ebi.ac.uk/ega - contact person: Rick Jansen, email: ri.jansen@ggzingeest.nl). Individual-level genotypes are not publicly available to ensure participant privacy, but access and permission can be requested from the BIOS consortium (https://www.bbmri.nl/ acquisition-use-analyze/bios). Summary statistics on the whole-blood gene expression, cell count imputation and expression-age associations are available from the BBMRI-NL atlas (http://bbmri.researchlumc.nl/atlas/). For the scRNA-seq data, please refer to [20].

\section{Ethics approval and consent to participate}

Written informed consent was obtained previously from all participants of the LL, LLS, NTR and RS biobanks in accordance with the ethical and institutional regulations. The LL study was approved by the Medical Ethics committee of the University Medical Centre Groningen (METC UMCG) document number METC UMCG LLDEEP: M12.113965) [11]. The study protocol for LLS was approved by the Medical Ethical committee of the Leiden University Medical Center (METC-LDD) before the start of the study [12]. The NTR study protocol was approved by Central Ethics Committee on Research Involving Human Subjects of the VU University Medical Center (CCMO), Amsterdam, an Institutional Review Board certified by the US Office of Human Research Protections (IRB number IRB2991 under Federal-wide Assurance-3703; IRB/institute codes, NTR 03-180) [13]. The RS was approved by the Medical Ethics Committee of the Erasmus MC (Erasmus MC MERC, registration number MEC 02.1015) and by the Dutch Ministry of Health, Welfare and Sport (Population Screening Act WBO, license number 1071272159521-PG) [14]. According to the METc UMCG, the study described in this manuscript is not a clinical research with human subjects as meant in the Medical Research involving Human Subjects Act (WMO). Therefore, no additional WMO approval of the study was required.

\section{Consent for publication}

Not applicable.

\section{Competing interests}

The authors declare no conflict of interest.

\section{Author details}

${ }^{1}$ Department of Genetics, University Medical Centre Groningen, Groningen, The Netherlands. '2Department of Biological Psychology, Netherlands Twin Register, Amsterdam Public Health research institute and Amsterdam Neuroscience, Vrije Universiteit Amsterdam, Amsterdam, The Netherlands. ${ }^{3}$ Department of Epidemiology, Erasmus University Medical Centre, Rotterdam, The Netherlands. ${ }^{4}$ Department of Biomedical Data Sciences, Leiden University Medical Center, Leiden, The Netherlands.

\section{Received: 23 June 2020 Accepted: 22 December 2020}

Published online: 15 March 2021

\section{References}

1. López-Otín C, Blasco MA, Partridge L, Serrano M, Kroemer G. The hallmarks of aging. Cell. 2013;153:1194.

2. Butler RN, Miller RA, Perry D, Carnes BA, Williams TF, Cassel C, et al. New model of health promotion and disease prevention for the 21 st century. BMJ. 2008;337:149-50

3. Peters MJ, Joehanes R, Pilling LC, Schurmann C, Conneely KN, Powell J, et al. The transcriptional landscape of age in human peripheral blood. Nat Commun. 2015;6.

4. Lin H, Lunetta KL, Zhao Q, Mandaviya PR, Rong J, Benjamin EJ, et al. Whole blood gene expression associated with clinical biological age. Journals Gerontol Ser A. 2019;74:81-8.

5. Palmer C, Diehn M, Alizadeh AA, Brown PO. Cell-type specific gene expression profiles of leukocytes in human peripheral blood. BMC Genomics. 2006;7:115.

6. Lin Y, Kim J, Metter EJ, Nguyen H, Truong T, Lustig A, et al. Changes in blood lymphocyte numbers with age in vivo and their association with the levels of cytokines/cytokine receptors. Immun Ageing. 2016;13.

7. Shaw AC, Goldstein DR, Montgomery RR. Age-dependent dysregulation of innate immunity. Nat Rev Immunol. 2013;13:875-87.

8. Solana R, Pawelec G, Tarazona R. Aging and innate immunity. Immunity. 2006;24:491-4.

9. Aguirre-Gamboa R, Joosten I, Urbano PCM, van der Molen RG, van Rijssen E, van Cranenbroek $B$, et al. Differential effects of environmental and genetic factors on T and B cell immune traits. Cell Rep. 2016;17:2474-87. 
10. Melé M, Ferreira PG, Reverter F, DeLuca DS, Monlong J, Sammeth M, et al. The human transcriptome across tissues and individuals. Science (80- ). 2015;348:660-5.

11. Tigchelaar EF, Zhernakova A, Dekens JAM, Hermes G, Baranska A, Mujagic Z, et al. Cohort profile: LifeLines DEEP, a prospective, general population cohort study in the northern Netherlands: study design and baseline characteristics. BMJ Open. 2015;5.

12. Schoenmaker M, de Craen AJM, de Meijer PHEM, Beekman M, Blauw GJ, Slagboom PE, et al. Evidence of genetic enrichment for exceptional survival using a family approach: the Leiden longevity study. Eur J Hum Genet. 2006;14:79-84

13. Willemsen G, De Geus EJC, Bartels M, Van Beijsterveldt CEMT, Brooks Al, Estourgie-van Burk GF, et al. The Netherlands twin register biobank: a resource for genetic epidemiological studies. Twin Res Hum Genet. 2010;13: 231-45

14. Ikram MA, Brusselle G, Ghanbari M, Goedegebure A, Ikram MK, Kavousi M, et al. Objectives, design and main findings until 2020 from the Rotterdam study. Eur J Epidemiol. 2020;35:483-517.

15. Aguirre-Gamboa R, de Klein N, di Tommaso J, Claringbould A, Võsa U, Zorro $M$, et al. Deconvolution of bulk blood eQTL effects into immune cell subpopulations. bioRxiv. 2019;548669.

16. Quinn KM, Fox A, Harland KL, Russ BE, Li J, Nguyen THO, et al. Age-related decline in primary CD8+ T cell responses is associated with the development of senescence in virtual memory CD8+ T cells. Cell Rep. 2018; 23:3512-24.

17. Van Rooij J, Mandaviya PR, Claringbould A, Felix JF, Van Dongen J, Jansen R, et al. Evaluation of commonly used analysis strategies for epigenome- a nd transcriptome-wide association studies through replication of large-scale population studies. Genome Biol. 2019;20.

18. Carroll RJ, Ruppert D. Transformation and weighting in regression. CRC Press. 1988;30.

19. Loguinov AV, Mian IS, Vulpe CD. Exploratory differential gene expression analysis in microarray experiments with no or limited replication. Genome Biol. 2004:5:R18

20. Van Der Wijst MGP, Brugge H, De Vries DH, Deelen P, Swertz MA, Franke L. Single-cell RNA sequencing identifies celltype-specific cis-eQTLs and coexpression QTLs. Nat Genet. 2018;50:493-7.

21. Kuleshov MV, Jones MR, Rouillard AD, Fernandez NF, Duan Q, Wang Z, et al. Enrichr: a comprehensive gene set enrichment analysis web server 2016 update. Nucleic Acids Res. 2016;44:W90-7.

22. Le Blanc J, Lordkipanidzé M. Platelet function in aging. Front Cardiovasc Med. 2019:6.

23. Campbell RA, Franks Z, Bhatnagar A, Rowley JW, Manne BK, Supiano MA et al. Granzyme a in human platelets regulates the synthesis of Proinflammatory cytokines by monocytes in aging. J Immunol. 2018;200: 295-304.

24. Wiley CD, Liu S, Limbad C, Zawadzka AM, Beck J, Demaria M, et al. SILAC Analysis Reveals Increased Secretion of Hemostasis-Related Factors by Senescent Cells. Cell Rep. 2019;28:3329-3337.e5.

25. Simon LM, Edelstein LC, Nagalla S, Woodley AB, Chen ES, Kong $X$, et al. Human platelet microRNA-mRNA networks associated with age and gender revealed by integrated plateletomics. Blood. 2014;123:e37-45.

26. Davizon-Castillo P, McMahon B, Aguila S, Bark D, Ashworth K, Allawzi A, et al. TNF-a-driven inflammation and mitochondrial dysfunction define the platelet hyperreactivity of aging. Blood. 2019;134:727-40.

27. Tacutu R, Thornton D, Johnson E, Budovsky A, Di B, Craig T, et al. Human ageing genomic resources: new and updated databases. Nucleic Acids Res. 2018;46(D1):D1083-90.

28. Bastyr EJ, Kadrofske MM, Vinik Al. Platelet activity and phosphoinositide turnover increase with advancing age. Am J Med. 1990;88:601-6.

29. Zahavi J, Jones NAG, Leyton J, Dubiel M, Kakkar W. Enhanced in vivo platelet "release reaction" in old healthy individuals. Thromb Res. 1980;17: 329-36.

30. Vitale $G$, Pellegrino G, Vollery M, Hofland LJ. ROLE of IGF-1 system in the modulation of longevity: Controversies and new insights from a centenarians' perspective. Front Endocrinol (Lausanne). 2019;10.

31. Fontana $L$, Partridge $L$, Longo VD. Extending healthy life span-from yeast to humans. Science (80- ). 2010;328:321-6.

32. Van Der Wijst MGP, de Vries DH, Groot HE, Trynka G, Hon CC, Bonder MJ, et al. The single-cell eQTLGen consortium. Elife. 2020;9.
33. Regev A, Teichmann SA, Lander ES, Amit I, Benoist C, Birney E, et al. Science forum: the human cell atlas. Elife. 2017;6:e27041.

34. Davizon-Castillo P, Rowley JW, Rondina MT. Megakaryocyte and platelet Transcriptomics for discoveries in human health and disease. Arterioscler Thromb Vasc Biol. 2020;ATVBAHA-119.

35. Brandsma M, Baas F, Bakker P, Beem E, Boomsma D, Bovenberg J, et al. How to kickstart a national biobanking infrastructure - experiences and prospects of BBMRI-NL. Nor Epidemiol. 2012;21.

36. Zhernakova DV, Deelen P, Vermaat M, Van Iterson M, Van Galen M, Arindrarto $\mathrm{W}$, et al. Identification of context-dependent expression quantitative trait loci in whole blood. Nat Genet. 2017:49:139-45.

37. Francioli LC, Menelaou A, Pulit SL, Van Dijk F, Palamara PF, Elbers CC, et al. Whole-genome sequence variation, population structure and demographic history of the Dutch population. Nat Genet. 2014;46:818-25.

38. Robinson MD, Oshlack A. A scaling normalization method for differential expression analysis of RNA-seq data. Genome Biol. 2010;11:R25.

39. eQTL mapping analysis cookbook for RNA seq data - molgenis/ systemsgenetics Wiki - GitHub. https://github.com/molgenis/ systemsgenetics/wiki/eQTL-mapping-analysis-cookbook-for-RNA-seq-data.

40. van Buuren S, Groothuis-Oudshoorn K. mice: Multivariate imputation by chained equations in R. J Stat Softw. 2011;45:1-67.

41. Little RJA. Missing-data adjustments in large surveys. J Bus Econ Stat. 1988;6: 287-96.

42. Babyak MA. What you see may not be what you get: a brief, nontechnical introduction to overfitting in regression-type models. Psychosom Med. 2004:66:411-21.

43. Võsa U, Claringbould A, Westra H-J, Bonder MJ, Deelen P, Zeng B, et al. Unraveling the polygenic architecture of complex traits using blood eQTL metaanalysis. bioRxiv. 2018;447367.

44. Bates D, Mächler M, Bolker BM, Walker SC. Fitting linear mixed-effects models using Ime4. J Stat Softw. 2015;67:1-48.

45. Willer CJ, Li Y, Abecasis GR. METAL: fast and efficient meta-analysis of genomewide association scans. Bioinformatics. 2010;26:2190-1.

46. Stouffer SA, Suchman EA, DeVinney LC, Star SA, Williams RM Jr. Adjustment during Army life. PUP. 1949;1.

47. Satija R, Farrell JA, Gennert D, Schier AF, Regev A. Spatial reconstruction of single-cell gene expression data. Nat Biotechnol. 2015;33:495-502.

\section{Publisher's Note}

Springer Nature remains neutral with regard to jurisdictional claims in published maps and institutional affiliations.

Ready to submit your research? Choose BMC and benefit from:

- fast, convenient online submission

- thorough peer review by experienced researchers in your field

- rapid publication on acceptance

- support for research data, including large and complex data types

- gold Open Access which fosters wider collaboration and increased citations

- maximum visibility for your research: over $100 \mathrm{M}$ website views per year

At BMC, research is always in progress.

Learn more biomedcentral.com/submissions 